\title{
Connectivity-Based Parcellation of the Amygdala Predicts Social Skills in Adolescents with Autism Spectrum Disorder
}

\author{
Annika Rausch $^{1}$ - Wei Zhang ${ }^{2,3}$ - Christian F. Beckmann ${ }^{1,2,4} \cdot$ Jan K. Buitelaar $^{1,5}$. \\ Wouter B. Groen ${ }^{5} \cdot$ Koen V. Haak ${ }^{1,2}$
}

Published online: 8 November 2017

(C) The Author(s) 2017. This article is an open access publication

\begin{abstract}
Amygdala dysfunction plays a role in the social impairments in autism spectrum disorders (ASD), but it is unclear which of its subregions are abnormal in ASD. This study compared the volume and functional connectivity (FC) strength of three FC-defined amygdala subregions between ASD and controls, and assessed their relation to social skills in ASD. A subregion associated with the social perception network was enlarged in ASD $\left(\mathrm{F}_{1}=7.842, p=.008\right)$ and its volume correlated significantly with symptom severity (social skills: $r=.548, p=.009$ ). Posthoc analysis revealed that the enlargement was driven by the vmPFC amygdala network. These findings refine our understanding of abnormal amygdala connectivity in ASD and may inform future strategies for therapeutic interventions targeting the amygdalofrontal pathway.
\end{abstract}

Christian F. Beckmann, Jan K. Buitelaar, Wouter B. Groen and Koen V. Haak have contributed equally to this work.

Annika Rausch

a.rausch@donders.ru.nl

1 Department of Cognitive Neuroscience, Donders Institute for Brain, Cognition and Behaviour, Radboud University Medical Center Nijmegen, P. O. Box 9101, 6500 HB Nijmegen, The Netherlands

2 Donders Institute for Brain, Cognition and Behaviour, Centre for Cognitive Neuroimaging, Radboud University, 6525 EN Nijmegen, The Netherlands

3 Behavioural Science Institute, Radboud University, 6525 HR Nijmegen, The Netherlands

4 Centre for Functional MRI of the Brain (FMRIB), University of Oxford, OX3 9DU Oxford, UK

5 Karakter Child and Adolescent Psychiatry University Center, 6500 HB Nijmegen, The Netherlands
Keywords Amygdala - Autism spectrum disorder · Functional connectivity $\cdot$ Parcellation $\cdot$ Prefrontal
Abbreviations
SBN
Social brain network
cACC
Caudal anterior cingulated cortex
IOFC
Lateral orbitofrontal cortex
vmPFC
Ventromedial prefrontal cortex
AOF parcel
Amygdala 1OFC parcel
APF parcel
Amygdala vmPFC parcel
AAC parcel
Amygdala cACC parcel
$\mathrm{FC}_{\mathrm{AOF}-\mathrm{OFF}}$
Functional connectivity between $\mathrm{AOF}$ and 1OFC
$\mathrm{FC}_{\mathrm{APF}-\mathrm{vmPFC}}$
Functional connectivity between APF and vmPFC
$\mathrm{FC}_{\mathrm{AAC}-\mathrm{cACC}}$ Functional connectivity between AAC and cACC

\section{Introduction}

Autism spectrum disorders (ASD) are a group of neuro developmental disorders characterized by severe impairments of reciprocal social interaction, verbal and nonverbal communication, repetitive and stereotyped behaviors and abnormal sensory processes (American Psychiatric Association 2013; American Psychiatric Association 2000). The amygdala is thought to play a crucial role in the function of the 'social brain' in terms of being involved in social cognition, emotion recognition, socio-communicative perception and the regulation of emotional responses (Phelps and LeDoux 2005). The amygdala theory (AT) for ASD therefore hypothesizes that amygdala dysfunction underlies the social deficits seen in ASD (Baron-Cohen et al. 2000). In line with the AT's predictions, the amygdala as a 
whole shows aberrant structural growth trajectories, exhibits abnormal functional connectivity (FC), and is involved in impaired emotion recognition and over-reactivity to aversive stimuli (Bellani et al. 2013; Green et al. 2013; Harms et al. 2010). The amygdala is, however, a composite structure, and its three major nuclei-the laterobasal, superficial and centromedial nuclei-have many connections with a wide variety of cortical areas. Yet, little is known about which amygdala pathways within the 'social brain network' (SBN) are compromised in ASD.

In earlier work, we used three anatomically defined amygdala subdivisions as seeds for a FC strength analysis and demonstrated that alterations within the amygdala network in ASD can be traced down to specific amygdala subdivisions. This approach, however, reveals functional connections that are associated with each amygdala subdivision throughout the whole brain and is therefore not system specific. In addition, anatomically defined subdivisions do not respect functional boundaries and vice versa, and each of the anatomically-defined amygdala subdivisions maintains connections along multiple pathways that are associated with various cognitive functions. In the present study, we therefore aimed to assess the functional architecture of the amygdala in adolescents with ASD by parcellating the amygdala based on its FC with three cortical seeds that are specifically anchored within the system of 'social brain networks' (SBNs).

Based on resting-state MRI scan data of healthy adults, Bickart et al. (2010, 2012) characterized three major SBNs that involved the amygdala: a 'social perception' network, a 'social affiliation' network and a 'social avoidance' network. For the 'social perception' network, the amygdala exhibited the strongest FC with the lateral orbitofrontal cortex (IOFC). The primary focus of amygdala connectivity for the 'social affiliation' network was found in ventromedial prefrontal cortex (vmPFC). Caudal anterior cingulate cortex (cACC) showed the strongest connectivity with the amygdala within the 'social avoidance' network. Bickart et al. $(2010,2012)$ used these cortical seed regions to parcellate the amygdala into three functional parcels: the ventrolateral, medial, and dorsal amygdala, respectively. Furthermore, the FC strength of these central nodes within the 'social perception' and 'social affiliation' networks correlated positively with the diversity and number of friends (Bickart et al. 2010, 2012).

Here, we first identified the three cortical seed regions found by Bickart et al. in our own adolescent healthy controls, and used these to parcellate the amygdala in both healthy controls and adolescents with ASD. We then compared the size of their associated parcels between the ASD group to the healthy controls. In posthoc analysis, we investigated these between-group differences further by delineating the relationship between the functional volumes and their FC strength to the three associated cortical seeds.
Finally, we tested whether functional volume serves as a marker for social skills in ASD. Because the FC strength of the amygdala's 'vmPFC' and 'IOFC' parcels have been reported as a good predictor of social network size (Bickart et al. 2010, 2012), we hypothesized that the volumes of the amygdala parcels predict the severity of social symptoms and impairment in ASD.

\section{Methods and Materials}

\section{Participants}

Twenty-one adolescents with autistic disorder according to the DSM-IV criteria and 25 typically developing controls were enrolled in the study. Participants with ASD were recruited through Karakter, Child and Adolescent Psychiatry University Center, Nijmegen. The study (including the informed consent procedure and all information brochures) was approved by both the regional ethics committee (Commissie Mensgebonden Onderzoek Arnhem Nijmegen) and Karakter's review board. All participants provided verbal and written informed consent.

We only included participants with an intelligence quotient (full-scale IQ) of 80 or higher. Sixteen participants with ASD and 19 control participants under age of 18 completed the Wechsler Intelligence Scale for Children III (WISCIII) (Kort et al. 2002), while participants above age of 18 $(\mathrm{ASD}=5$, controls $=6)$ completed the Wechsler Adult Intelligence Scale III (WAIS-III) (Wechsler 2000). All participants also completed the short version of Edinburgh Handedness Inventory (Oldfield 1971).

All participants and their parents completed the Autism Spectrum Quotient (AQ) about themselves or their child respectively. The AQ is a validated measure of autism spectrum characteristics found within both the typical population and individuals with a diagnosis of ASD and thus provides a reliable measurement tool for the comparison of autistic traits between our ASD and control sample (Baron-Cohen et al. 2001).

Diagnoses of autistic disorder were based on a series of clinical assessments including a detailed developmental history, clinical observation, medical work-up and cognitive testing in a multidisciplinary team including a child psychiatrist and clinical psychologist. Diagnoses of autistic disorder was acquired with the Autism Diagnostic Interview-Revised (ADI-R) (Lord et al. 1994). We excluded those with ASD who had co-morbid psychiatric or neurological conditions including but not limited to attention deficit/hyperactivity disorder (ADHD), depressive disorder, schizophrenia, epilepsy or history of traumatic brain injury. None of the participants used medication. 
Table 1 Subject demographics

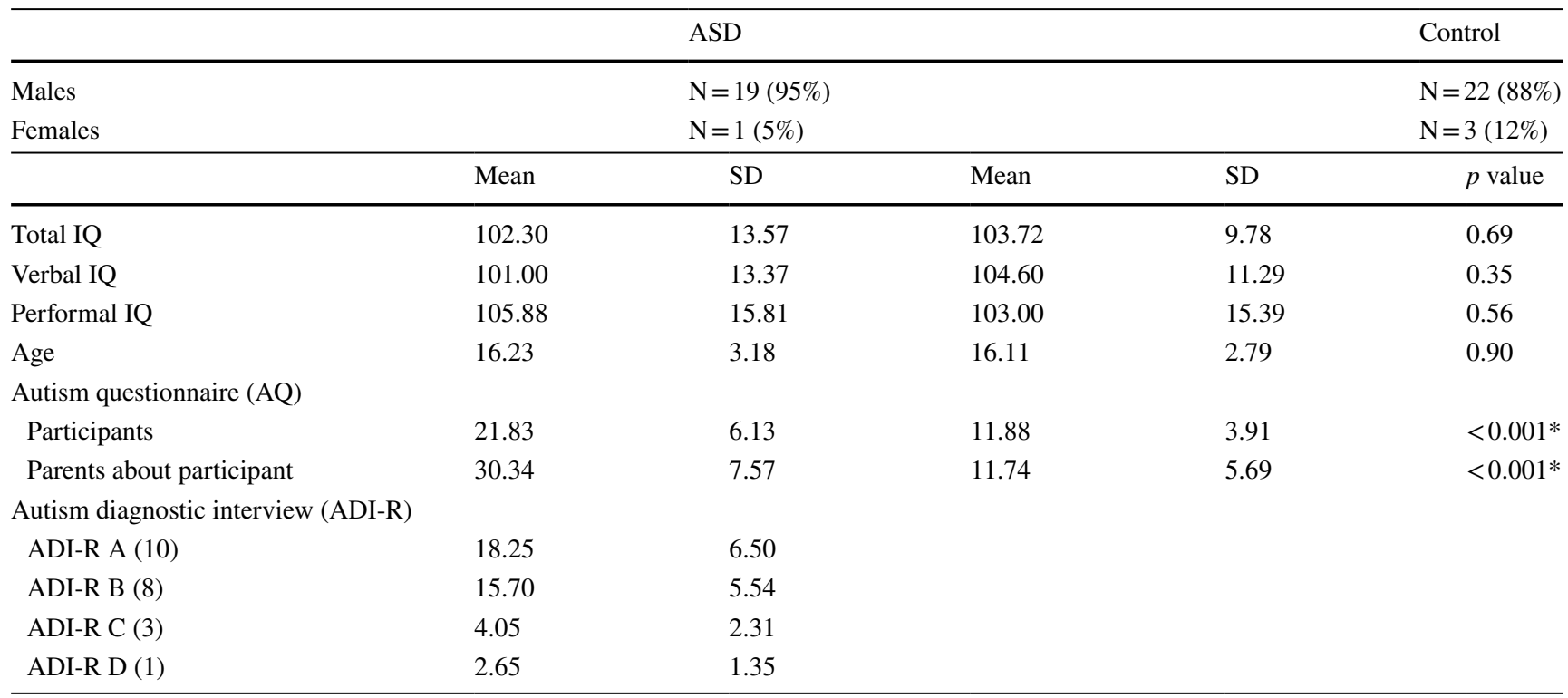

$p$ value $=p$ values indicate results for the independent t-test statistic. ADI-R (A) social interaction, (B) communication and language, $(\mathrm{C})$ restricted and repetitive behavior (D) age of onset criterium; ADI-R thresholds are shown in parentheses. Pearson chi-squared for group by gender was non-significant (value $=0.672, \mathrm{df}=1,2$-sided asymptotic $p=.412$ )

*Statistically significant

Controls were matched at the group level on age, sex, and handedness and verbal, performance and full-scale IQ scores (Table 1). We ruled out the presence of psychiatric co-morbidity in controls and verified that all participants scored within the normal range using the school-age version of Child Behavior Check List (CBCL/6-18) and Adult Behavior Check List (ABCL/18-59).

\section{Image Data Acquisition}

For each participant, we acquired MRI data at the Donders Institute for Brain, Cognition and Behaviour, Center for Cognitive Neuroimaging in Nijmegen, The Netherlands, using a 3 T Magnetom TIM Trio (Siemens, Erlangen, Germany) with a 32-channel head coil. The entire scanning session lasted approximately $45 \mathrm{~min}$. For each participant, we collected a T1-weighted whole-brain scan (magnetizationprepared rapid acquisition with gradient echo [MPRAGE], $\mathrm{TI}=1100 \mathrm{~ms}, \mathrm{TR}=2300 \mathrm{~ms}, \mathrm{TE}=3.03 \mathrm{~ms}$, flip angle $=8^{\circ}$, FOV $=256 \times 256 \times 192 \mathrm{~mm}^{3}$, voxel size $=1 \times 1 \times 1 \mathrm{~mm}^{3}$ ) and a resting-state scan using T2*-weighted dual-echo planar imaging $(E P I, T R=2510 \mathrm{~ms}$, TE $1=16 \mathrm{~ms}, \mathrm{TE} 2=36 \mathrm{~ms}$, flip angle $=83^{\circ}, \mathrm{FOV}=212 \times 212 \times 119 \mathrm{~mm}^{3}$, voxel size $=2 \times 2 \times 2.5 \mathrm{~mm}^{3}$, number of volumes $=400$, imaging bandwidth $=1814 \mathrm{~Hz} / \mathrm{px}$, grappa acceleration factor $=4$ ). Note that the usage of dual-echo imaging provides optimal sensitivity for BOLD imaging in both subcortical structures such as the amygdala and the neocortex (Poser et al. 2006). Participants were instructed to lie still within the scanner with their eyes open during the resting-state scan, while staying awake and focusing on a small white cross presented at the center of a projection screen. The first five volumes $(12.55 \mathrm{~s})$ were discarded to reduce magnetization equilibration effects. Gradient echo field mapping data were also acquired with identical geometry to the EPI data for EPI offresonance distortion correction $(\mathrm{TR}=1020 \mathrm{~ms}, \mathrm{TE} 1=10 \mathrm{~ms}$, TE2 $=12.46 \mathrm{~ms}$, flip angle $=90^{\circ}, \mathrm{FOV}=224 \times 224 \times 191$ $\mathrm{mm}^{3}$, voxel size $=3.5 \times 3.5 \times 2 \mathrm{~mm}^{3}$ ). All participants were able to familiarize themselves with scanner set-up and scanning procedure through rehearsal in a replicate (dummy) scanner before actual image acquisition.

We recorded participants' heartbeats using the scanner's built-in photoplethysmograph, placed on the right index finger. Respiration was measured with a pneumatic belt positioned at the level of the abdomen. To reduce the potential effects that heartbeat and respiration have on resting-state BOLD correlation studies (Birn et al. 2008; Chang et al. 2013), we used cardiac and respiratory phase regressors, as well as other nuisance regressors in the fMRI time series analysis.

\section{Preprocessing}

All image preprocessing and analyses were performed using FSL (FMRIB Software Library, http://fmrib.ox.ac. uk/fsl) (Smith et al. 2004). The following pre-statistical processes were applied to the fMRI data: non-brain removal using BET; rigid-body motion correction using 
MCFLIRT; high-pass temporal filtering (Gaussianweighted least-squares fitting with frequency cutoff point $=100 \mathrm{~s}$ ); correction of off-resonance geometric distortions in the EPI data using PRELUDE and FUGUE, using $\mathrm{B}_{0}$ field maps derived from the dual-echo gradient echo dataset; artifact removal based on probabilistic ICA (Independent Component Analysis) using MELODIC; spatial normalization to Montreal Neurological Institute (MNI152) $2 \mathrm{~mm}$ isotropic atlas space using BoundaryBased-Registration (BBR) and FNIRT and Gaussian filtering (FWHM $=6 \mathrm{~mm}$; see "Methods"). The dual-echo images ( $\mathrm{TE}=16$ and $\mathrm{TE}=36$ ) were combined by averaging both echo-times. We excluded 1 participant with ASD due to excessive head movement in terms of frame-wise displacement (Max. $F D=8.7 \mathrm{~mm}, \mathrm{M}_{\mathrm{fd}}=0.89 \mathrm{~mm}$ ), resulting in 20 datasets from the ASD group and 25 datasets from the control group for further analysis (Rausch et al. 2016). To rule out that differences in movement between the ASD and control group could contribute to the results, we calculated the mean value of frame-wise movement (i.e. the movement of one TR relative to previous TR) for each participant and compared it between the two groups. No group difference was found $\left(\mathrm{M}_{\mathrm{asd}}=0.10, \mathrm{SD}_{\mathrm{asd}}=0.10\right.$; $\left.\mathrm{M}_{\mathrm{ctrl}}=0.07, \mathrm{SD}_{\mathrm{ctrl}}=0.42 ; t_{43}=0.312, p=.757\right)$.

\section{Controlling for Structured Noise}

Our preprocessing stream included several steps to limit the influence of structured noise, such as motion artifacts (Power et al. 2012), heartbeat (Chang et al. 2013), and respiration (Birn et al. 2008). First, we conducted manual ICA-based artifact removal (Rausch et al. 2016). The first author visually inspected all the independent component maps for each participant to identify noise components based on the spatial layout of the component maps and the power spectra of the associated time-series (Kelly Jr et al. 2010). We applied non-aggressive denoising with FSL's fsl_regfilt, i.e. only variance that was uniquely related to the components labeled as noise component (approx. 70\% of components) was removed.

After ICA-based noise removal and further preprocessing, we conducted nuisance regression modeling the potential effect from motion and physiological noise on the resting-state fMRI data. Specifically, we included six rigid-body parameters and the eigenvariate of signals over the entire white matter and the CSF in our GLM. Moreover, we calculated ten cardiac phase regressors, ten respiratory phase regressors and six other nuisance regressors including heart rate fluctuation, heart rate variability, respiration raw data averaged per TR, respiratory amplitude in $9 \mathrm{~s}$ window, respiratory frequency in $9 \mathrm{~s}$ window and the frequency times amplitude of respiration (averaged per TR) that are derived from the RETROICOR method (Glover et al. 2000).

\section{Defining Cortical Seed Points}

We first extracted the mean time series from a bilateral amygdala mask (using FWHM $=1 \mathrm{~mm}$ Gaussian filtered functional images for the amygdala) and calculated its correlations with every voxel in the rest of the brain (using $\mathrm{FWHM}=6 \mathrm{~mm}$ Gaussian filtered functional images for the whole brain) (Fig. 1a). Different smoothing kernels for the amygdala were used, because the width of the amygdala filter should be tailored to the parcel size between-group differences we expect to see (Rosenfeld 1976). The bilateral amygdala ROI was defined using probabilistic maps from the Harvard-Oxford Subcortical Structural Atlas available for FSL, limited to voxels that had $25 \%$ or greater probability of being labeled as the amygdala (left: $3392 \mathrm{~mm}^{3}$, right: $3888 \mathrm{~mm}^{3}$ ). We then identified peak voxels from the $1-\mathrm{p}$ statistical correlation map $(p=.05)$ between the amygdala and areas within the boundaries of probabilistic vmPFC, IOFC and cACC maps within each hemisphere (Harvard-Oxford Structural Atlas) and created a $3 \mathrm{~mm}$ cortical sphere around the resulting six coordinates $(\mathrm{cACC}= \pm 2$, $-2,38 ; 1 \mathrm{OFC}= \pm 40,28,-18 ; \operatorname{vmPFC}= \pm 2,46,-18)$. We then combined the coordinates to create three bilateral seed regions (IOFC, vmPFC and cACC), known to be involved in adaptive social behavior (Fig. 1b). The definition of the cortical seed regions was based solely on the control group.

\section{Parcellation of the Amygdala}

We then parcellated the amygdala based on its FC with the three cortical seed regions. FSL's SBCA was used to calculate the correlation between the mean time series of the voxels in each cortical seed and the time series of every voxel within the bilateral amygdala, corrected for the mean time series within the other two cortical seed regions. Thus, one single-subject partial correlation map of the amygdala for each hub within its network was obtained, which represented their unique connectivity with the amygdala. The partial correlation maps were $r$-to- $Z$ transformed and each voxel was assigned to the network with the maximum $Z$-value. As a result, the amygdala was parcellated into three functional parcels: one parcel defined by maximal $\mathrm{FC}$ with the IOFC, one defined by maximal FC with the vmPFC, and one defined by maximal FC with cACC, which we will refer to as 'AOF', 'APF', and 'AAC' parcels respectively (Fig. 1c).

\section{Functional Amygdala Network Volume Analysis}

To test whether the volume of the amygdaloid parcels differed between diagnostic groups, we extracted the three 


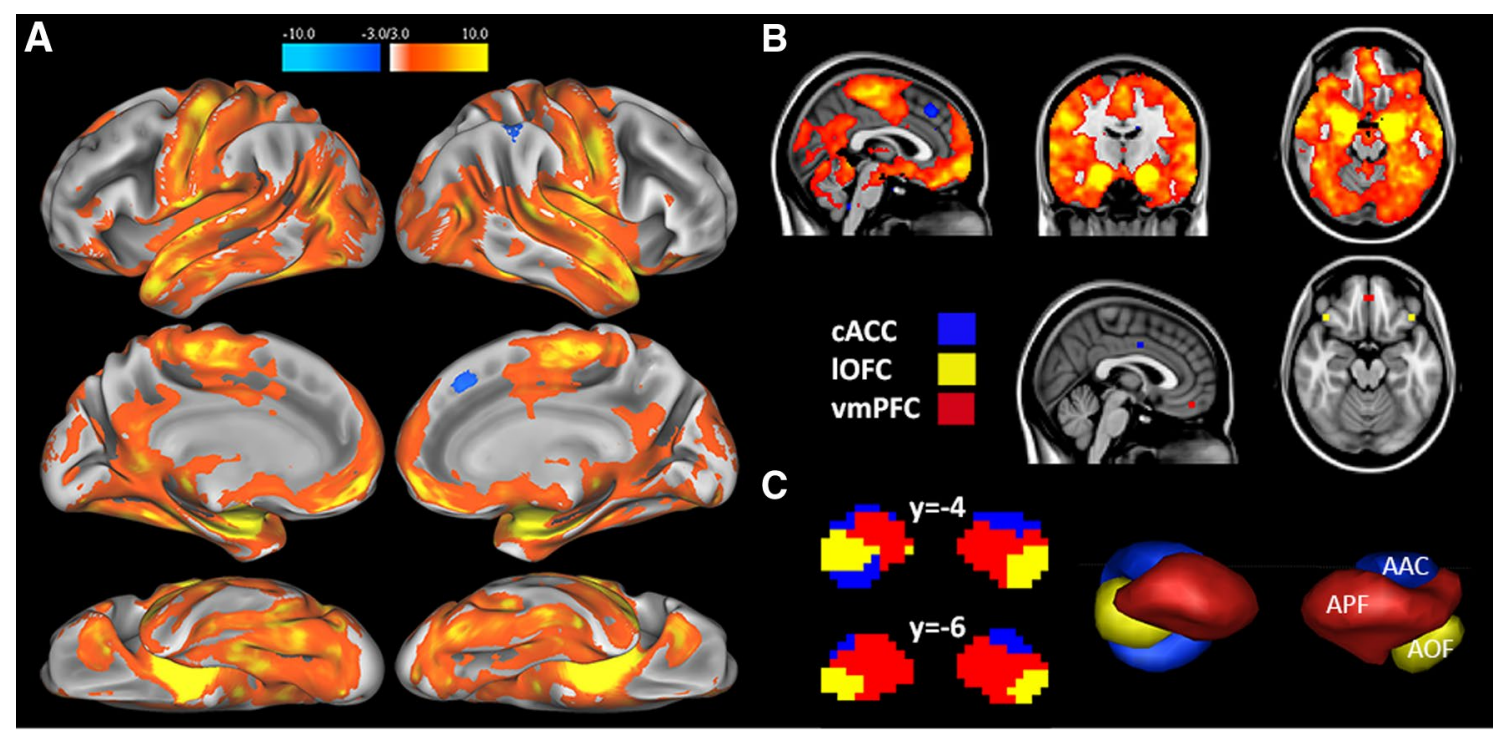

Fig. 1 Spatial distribution of the three amygdalo-cortical networks from which cortical seed coordinates were defined and amygdala parcellation in controls. Panel a shows the t statistics for the amygdalo-cortical activation map from lateral, medial and ventral view on an inflated brain. Panel b shows the same from the coronal, saggital and axial view on a T1 MNI152 $2 \mathrm{~mm}$ template brain in radiologic convention in the upper section. The lower section shows the cortical seeds positioned in the cACC, $1 \mathrm{OFC}$ and $\operatorname{vmPFC}(\mathrm{cACC}= \pm 2$,

parcel volumes per subject and conducted second-level analysis in SPSS using ANCOVA (separate dependent variables: AOF, APF and AAC volume; fixed factors: diagnostic group; covariates: age and grey matter volume) (IBM Corp 2016). One participant was identified as an outlier using the standard definition of outliers as implemented in SPSS due to extreme values of the AAC parcel volume $\left[\mathrm{N}_{\mathrm{ASD}}=19 ; \mathrm{N}_{\mathrm{Ctr}}=25\right.$; i.e., values outside of 1.5 times the interquartile range as implemented in SPSS 23 (IBM Corp 2016)] and excluded from the AAC parcel volume analysis, though removing this outlier did not change the statistical significance of the results. The data was normally distributed within the control and the ASD group [Shapiro-Wilk statistics: $\operatorname{ASD}_{\mathrm{AAC}}(\mathrm{df}=19, p=.081) ; \operatorname{ASD}_{\mathrm{AOF}}(\mathrm{df}=20, p=.412)$; $\operatorname{ASD}_{\mathrm{APF}}(\mathrm{df}=20, p=.222) ; \mathrm{CTR}_{\mathrm{AAC}}(\mathrm{df}=25, p=.950)$; $\left.\mathrm{CTR}_{\mathrm{AOF}}(\mathrm{df}=25, p=.610) ; \mathrm{CTR}_{\mathrm{APF}}(\mathrm{df}=25, p=.278)\right]$.

\section{FC Strength Posthoc Analysis}

After assessing the FC volume of each parcel in ASD and controls, we examined the FC strength between the AOF, $\mathrm{APF}$ and AAC parcels with their corresponding cortical seeds in controls and ASD, which we will refer to as ' $\mathrm{FC}_{\mathrm{AOF}-\mathrm{IOFC}}$ ', ' $\mathrm{FC}_{\mathrm{APF}-\mathrm{vmPFC}}$ ' and ' $\mathrm{FC}_{\mathrm{AAC}-\mathrm{cACC}}$ ' strength respectively. To assess the direction of FC strength differences between both diagnostic groups, we extracted for each parcel the voxel-wise (Fisher $r$-to-Z transformed)
$-2,38 ; 10 F C= \pm 40,28,-18 ; \operatorname{vmPFC}= \pm 2,46,-18)$. Cortical seeds were defined based on the full correlation maps with the entire amygdala in the control group. Panel c shows a visualization of the FC parcellation of the amygdala in the control group. Each voxel was assigned to the network with the maximum $Z$-value at the grouplevel. The left section shows the parcellation in $2 \mathrm{~d}$ slices from two axes and the right section shows the parcellation using $3 \mathrm{~d}$ rendering

partial correlations with their associated cortical target, and averaged these across all voxels in that parcel using SBCA [seed based correlation analysis (O'reilly et al. 2009)]. As we want to investigate those voxels that drove the differences between the ASD and control group, and because we do not expect to find any differences in FC strength in regions that belong to the same parcel in both groups, we used the average AOF, APF, and AAC parcels as defined within the control group as ROIs in this analysis. Note that the better parcel definition in controls as compared to the ASD group by itself might cause a bias towards higher FC strength in the control group. However, as the influence of neighboring parcels is regressed out using partial correlation analysis, and because our analysis approach therefore corrects for mixed signals between one parcel and its neighboring parcels in the ASD group, we thus corrected for the poorer parcel definition in the ASD group. Between-group comparison was carried out in SPSS using ANCOVA (separate dependent variables: The $\mathrm{FC}_{\mathrm{AOF}-1 \mathrm{OFC}}, \mathrm{FC}_{\mathrm{APF}-\mathrm{vmPFC}}$ and $\mathrm{FC}_{\mathrm{AAC}-\mathrm{cACC}}$ strength; fixed factors: diagnostic group; covariates: age). Four participants were excluded in the $\mathrm{FC}_{\mathrm{AOF}-1 \mathrm{OFC}}$ strength analysis and one participant was excluded from the $\mathrm{FC}_{\mathrm{APF}-\mathrm{vmPFC}}$ strength due to extreme values $\left(\mathrm{FC}_{\mathrm{AOF}-1 \mathrm{OFC}}\right.$ strength: $\mathrm{N}_{\mathrm{ASD}}=19 ; \mathrm{N}_{\mathrm{Ctr}}=22 ; \mathrm{FC}_{\mathrm{APF}-\mathrm{vmPFC}}$ strength: $\mathrm{N}_{\mathrm{ASD}}=20$; $\mathrm{N}_{\mathrm{Ctr}}=24$; i.e., according to the $1.5 \times \mathrm{IQR}$ rule), though this did not change the statistical significance of the results. 
The data was normally distributed within the control and the ASD group [Shapiro-Wilk statistics: ASD FC AAC-cACC $(\mathrm{df}=20, p=.143) ; \mathrm{ASD} \mathrm{FC}_{\mathrm{AOF}-1 \mathrm{OFC}}(\mathrm{df}=19, p=.808)$; $\operatorname{ASD~FC}_{\mathrm{APF}-\mathrm{vmPFC}}(\mathrm{df}=20, p=.992)$; CTR FC $\mathrm{AAC}-\mathrm{cACC}$ $(\mathrm{df}=25, p=.752) ; \mathrm{CTR} \mathrm{FC}_{\mathrm{AOF}-1 \mathrm{OFC}}(\mathrm{df}=22, p=.879)$; CTR $\left.\mathrm{FC}_{\mathrm{APF}-\mathrm{vmPFC}}(\mathrm{df}=24, p=.538)\right]$.

\section{Results}

\section{Connectivity-Based Parcellation of the Amygdala in ASD Versus Controls}

We first aimed to assess the functional architecture of the central hubs within the amygdala SBNs in ASD and controls. The amygdala was parcellated into three functional parcels (AOF, APF and AAC) based on its FC with each of the three cortical seeds (IOFC, vmPFC and cACC). Figure 1c shows the group-level parcellation for the controls, which corresponds well with the previous functional parcellation in healthy adults described by Bickart et al. (2012). The AAC, AOF and APF parcels yielded a mean (SD) volume across controls of $2031 \mathrm{~mm}^{3}\left(879.3 \mathrm{~mm}^{3}\right), 2108.8 \mathrm{~mm}^{3}$ $\left(883.4 \mathrm{~mm}^{3}\right)$ and $3140.2 \mathrm{~mm}^{3}\left(1100 \mathrm{~mm}^{3}\right)$ respectively. In the ASD group the mean parcel volume was $1576 \mathrm{~mm}^{3}$ $\left(1244.2 \mathrm{~mm}^{3}\right)$ in AAC, $3059.6 \mathrm{~mm}^{3}\left(1338.7 \mathrm{~mm}^{3}\right)$ in AOF and $2454.8 \mathrm{~mm}^{3}\left(1231 \mathrm{~mm}^{3}\right)$ in APF. To verify that this functional parcellation is based on meaningful signals, we confirmed that all parcels in both groups yielded significant FC with the corresponding cortical seeds (all $p<.001$ for both groups). Note however that the significance of these functional connections is to be expected given that we

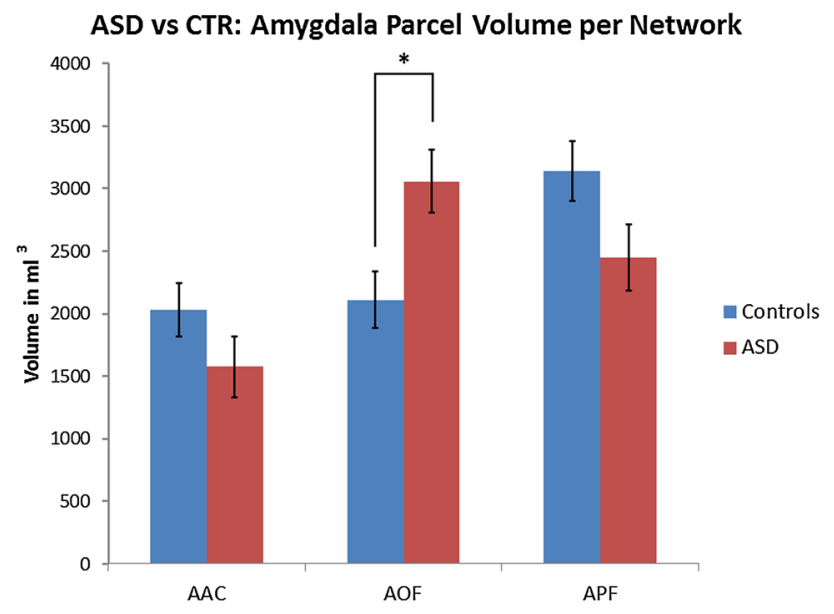

Fig. 2 Amygdala parcel volume estimated marginal means in ASD and controls. The AOF parcel (middle) was significantly $(p=.008)$ increased in ASD (red) compared to controls (blue), while there was a non-significant trend of decreased APF (right) and AAC (left) parcels in ASD compared to controls. Error-bars represent the standard error. (Color figure online) defined the cortical seeds by selecting those cortical locations as seed areas based on significant connectivity with the entire amygdala.

We then compared the volume of the parcels between the ASD group and controls for each parcel individually. We found a significant increase in AOF parcel volume in the ASD group $\left(\mathrm{F}_{1}=7.842, p=.008\right.$; age and total grey matter volume corrected) and a trend toward decreased APF volume $\left(\mathrm{F}_{1}=3.794, p=.058\right)$ and AAC volume $\left(\mathrm{F}_{1}=1.990\right.$, $p=.166$ ) (Fig. 2).

\section{Relationship Between Parcel Volume and Symptom Severity}

To investigate whether the larger AOF parcel in ASD is associated with ASD social symptoms and its severity, we conducted correlation analysis (corrected for age and total grey matter volume) between the volume of AOF parcel and the social skills subdomain of the AQ. We found that AOF volume predicted symptom severity in the social skills subdomain ( $r=.548, \mathrm{df}=16, p=.009)$ of the ASD group.

\section{FC Strengths Posthoc Analysis Within Networks}

To understand the underlying biological mechanism that drives the volume changes in the ASD group, we assessed their $\mathrm{FC}$ strength (' $\mathrm{FC}_{\mathrm{AAC}-\mathrm{CACC}}$ ', 'FC $\mathrm{AOF}_{\mathrm{AOFC}}$ ' and ' $\mathrm{FC}_{\mathrm{APF}-\mathrm{vmPFC}}$ ') in a posthoc analysis. Our functional volume parcellation approach is based on a winner-takes-all method, which assigned one amygdala voxel to one central hub within one SBN. Thus, with this method, we reveal a FC map that is based on the strongest connectivity value with one SBN as compared to the other network hubs. By itself, this map does not contain information about the actual strength of connection with the winning SBN as it is based on the relative strength between networks within a diagnostic group. The FC strength measure, on the other hand, enables us to investigate whether the $\mathrm{AOF}$ volume group difference was driven by abnormalities (i.e. FC strength increases) originating directly within its corresponding social perception SBN, or whether the $\mathrm{AOF}$ volume group difference was driven by alterations (i.e. FC strength decreases) within one or both of the neighboring SBN's. Therefore, we assessed whether the increase in volume of the AOF parcel could be the result of two different mechanisms: (1) increased $\mathrm{FC}_{\mathrm{AOF}-\mathrm{IOFC}}$ strength in the ASD group, or (2) decreased FC strengths in one or two of the other networks hubs in the ASD group. To disambiguate which of these two is driving the AOF volume difference, we tested the direction of FC strength change for each SBN. The results show a decrease in $\mathrm{FC}_{\mathrm{APF}-\mathrm{vmPFC}}$ strength $\left(\mathrm{F}_{1}=8.596, p=.005\right)$, but no between-group effects in $\mathrm{FC}_{\mathrm{AAC}-\mathrm{cACC}}$ strength $\left(\mathrm{F}_{1}=2.022, p=.162\right)$ and $\mathrm{FC}_{\mathrm{AOF}-\mathrm{IOFC}}$ strength $\left(\mathrm{F}_{1}=0.170, p=.682\right)$ (Fig. 3). These results indicate 


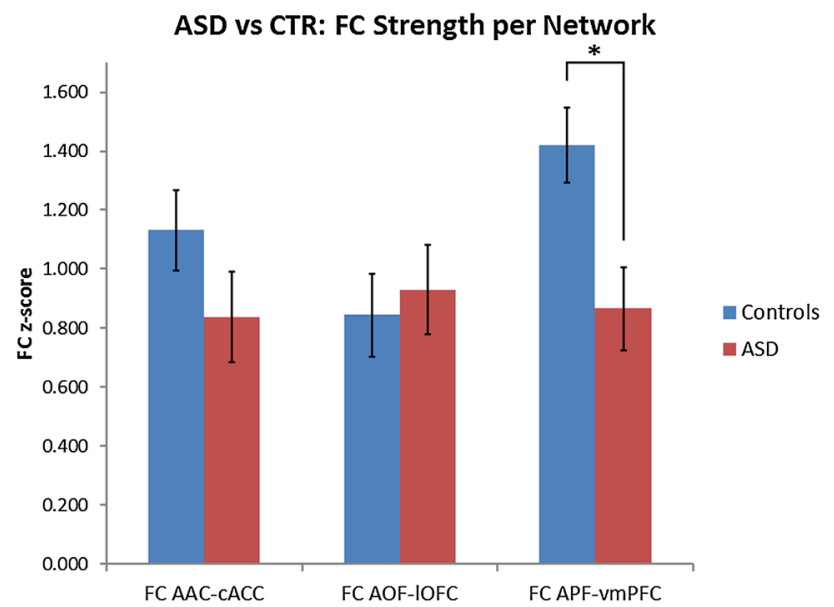

Fig. 3 FC strength per parcel in the ASD group and controls. The $\mathrm{FC}_{\mathrm{APF}-\mathrm{vmPFC}}$ (right) FC strength was significantly decreased in ASD (red) compared to controls (blue), with a non-significant decrease in $\mathrm{FC}_{\mathrm{AAC}-\mathrm{cACC}}$ (left) and increase in $\mathrm{FC}_{\mathrm{AOF}-\mathrm{OOFC}}$ (middle) $\mathrm{FC}$ strength in ASD compared to controls. Error-bars represent the standard error. (Color figure online)

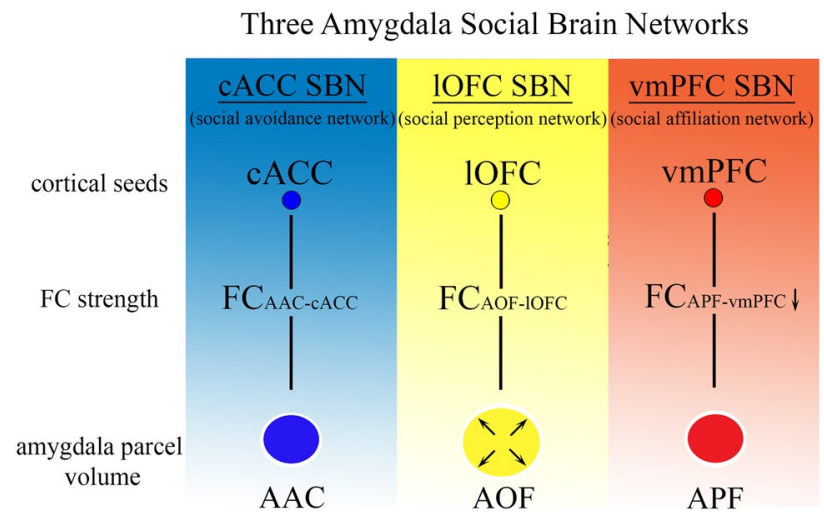

Fig. 4 Schematic overview of the three amygdala SBNs. The blue, yellow and red panels show the cortical seeds, FC strength parameters and amygdala parcel volume parameters per amygdala social brain network $(\mathrm{SBN})$. Arrows indicate increases or decreases in FC within an SBN. In the ASD group, AOF parcel volume was increased in $\mathrm{ASD}$, while $\mathrm{FC}_{\mathrm{APF}-\mathrm{vmPFC}}$ strength was reduced. (Color figure online)

that the increase in volume of the AOF parcel originated from the APF parcel, because the FC strength between AOF and IOFC is constant between ASD and controls, while the FC strength between APF and vmPFC was lower for the ASD group compared with controls (Fig. 3).

To investigate whether the reduced $\mathrm{FC}_{\mathrm{APF}-\mathrm{vmPFC}}$ strength in ASD is associated with ASD social symptoms and its severity, we conducted a correlation analysis (corrected for age) between the $\mathrm{FC}_{\mathrm{APF}-\mathrm{vmPFC}}$ strength and the social skills subdomain of the AQ, which was not significant $(r=-.087$, $\mathrm{df}=17, p=.362)$. Furthermore, though we only found a weak trend towards reduced $\mathrm{FC}_{\mathrm{AAC}-\mathrm{cACC}}$ strength, the $\mathrm{AOF}$ parcel enlargements may in part relate to the marginally significant AAC parcel reductions. Therefore, the combined $\mathrm{FC}_{\mathrm{APF}-\mathrm{vmPFC}}$ and $\mathrm{FC}_{\mathrm{AAC}-\mathrm{cACC}}$ strength might explain more variance of the social symptoms scores in ASD than the $\mathrm{FC}_{\mathrm{APF}-\mathrm{vmPFC}}$ strength alone. Thus, we assessed whether their combined FC strength could predict social AQ scores using linear regression analysis (corrected for age). The result was non-significant $\left(\mathrm{F}_{3}=2.082, p=.143\right)$. Figure 4 contains a schematic overview of the main results.

\section{Discussion}

We assessed the functional architecture of the amygdala in adolescents with ASD by parcellating the amygdala based on its FC with three cortical seeds (cACC, 1OFC and vmPFC) that are anchored within known SBNs: the social avoidance network, the social perception network and the social affiliation network. Three functional parcels were created (AAC, AOF and APF) based on its FC with each of the three cortical seeds respectively. We found a significant enlargement of the AOF parcels in the ASD group, while there was a trend toward decreased volume of the other two parcels in ASD, especially of the APF parcel. We assessed the clinical relevance of our marker, and found that increased AOF parcel volume predicted impairments in social skills in the ASD group. In posthoc analysis, we found that the increase of the AOF parcel came at the cost of the APF parcel, as indicated by a decrease in $\mathrm{FC}_{\mathrm{APF}-\mathrm{vmPFC}}$ strength.

Our results align well with and extend earlier findings showing that especially the $1 \mathrm{OFC}$ and vmPFC amygdala SBNs predicted social outcome in a healthy control sample (Bickart et al. 2012). All three affective networks are associated with generating appropriate adaptive social behavior and are known to work together closely (Bickart et al. 2014). We show for the first time that ASD is associated with FC abnormalities of the IOFC and vmPFC amygdala pathways, which are the central hubs of social perception and social affiliation network functionality. The AOF parcel roughly corresponds to the ventrolateral subregion containing the laterobasal nucleus of the amygdala and the APF parcel to the medial subregion containing the superficial nucleus according to the probabilistic cytoarchitectonically defined Jülich atlas (Amunts et al. 2005), which are associated with perceptual input processing of the amygdala (Rausch et al. 2016). Although the AAC parcel roughly corresponds to the dorsal amygdala containing the centromedial amygdala nucleus, Fig. 1c indicates hemispheric lateralization related to the AAC parcel. More specifically, the right AAC extends into the ventrolateral subregion, which has been assigned to the AOF parcel in the left hemisphere. The literature on 
lateralization effects on subregion level in the amygdala is however sparse, and it is therefore unclear what the functional implications of this lateralization effect are (Gläscher and Adolphs 2003; Gorka et al. 2017; McMenamin and Marsolek 2013). The AAC did not show any group effects in the FC strength analysis or parcel volume in our study. Our findings therefore may be in line with an earlier study investigating abnormalities in three anatomically defined amygdala subdivisions (the laterobasal, superficial and centromedial nuclei) that implicated amygdala under-connectivity between the superficial and laterobasal nuclei and cortex (Rausch et al. 2016).

Given the inhibitory relationship between the vmPFC and the amygdala in the literature (Motzkin et al. 2015), our findings of reduced $\mathrm{FC}_{\mathrm{APF}-\mathrm{vmPFC}}$ strength may point toward weaker inhibitory connections of the VmPFC amygdaloid circuit in ASD. Our posthoc FC strength analysis also demonstrates that a potential lack of inhibition from the vmPFC onto the amygdala in ASD is not linked to significantly increased FC strength of the 1OFC amygdaloid network, because the $\mathrm{FC}_{\mathrm{APF}-\mathrm{vmPFC}}$ strength decrease was not accompanied by a significant increase in $\mathrm{FC}_{\mathrm{AOF}-\mathrm{IOFC}}$ strength. However, our results might indicate that a potential lack of inhibition from the vmPFC might be driving a weak - but not significant-increase of $\mathrm{FC}_{\mathrm{AOF}-\mathrm{OFC}}$ strength in ASD, that is spanning a significantly larger area in the ASD group as compared to controls. Recent findings suggest that abnormalities in FC strength are rather characterized by a diffuse distribution of FC in ASD (Hahamy et al. 2015). In other words, the autistic brain may have idiosyncratic FC patterns, which cannot be identified in terms of a "common spatial locus" of abnormal FC strength changes. Instead, the areas in which the $\mathrm{FC}$ abnormalities occur, might be characterized by different FC between ASD subjects, which might partially explain diffuse and widespread FC changes in ASD. This suggests that ASD is not a disorder of unique abnormal loci per se, but rather a problem of the functional specialization as compared to controls. Therefore, because our functional volume measures provide a quantification of FC that is independent of a "common spatial locus" of abnormal activation within the amygdala (yet tied to specific functionality), our functional volume measure might indicate that alterations of the social perception 1OFC amygdala network, are characterized by abnormal FC distribution.

We also assessed how well our FC markers predict social skills based on the AQ social subdomain in the ASD group. Since the increase of the AOF volume may be a consequence of decreased $\mathrm{FC}_{\mathrm{APF}-\mathrm{vmPFC}}$ strength, the correlation between decreased $\mathrm{FC}_{\mathrm{APF}-\mathrm{vmPFC}}$ strength and social skills was tested, but was not significant. However, we were able to relate our findings of increased $\mathrm{AOF}$ volume to social skills. Because the increase in $\mathrm{AOF}$ volume appears to be a consequence of decreased $\mathrm{FC}_{\mathrm{APF}-\mathrm{vmPFC}}$ strength, and because the $\mathrm{AOF}$ volume significantly predicted social skills in the ASD group, it might be surprising that we did not find significant negative relationships between social skills and the FC strength of the APF (and/or ACC) parcel(s). The most parsimonious explanation for this apparent inconsistency is that parcel volume and FC strength measure different things and that the first better probes the underlying pathology than the latter. A parcel's volume is dependent on the number of voxels that exhibited maximum partial correlation with that parcel's cortical target. Maximum partial correlation can be achieved by very small correlation differences, so for instance a relatively large parcel volume can be due to having many voxels with very small correlation differences. As such, differences in parcel volume can be great while the difference in average FC strength is very small. In other words, parcel volume, though derived from FC strength estimates, does not have to follow the same pattern as the average FC strength. The fact that parcel volume better predicts social skills than average FC strength can further be taken to imply that the underlying pathology can be attributed to a large number of voxels (neurons) that exhibit an abnormal balance in terms of its connectivity with the three cortical targets with only subtle alterations in the strength of these connections. Therefore, our functional parcel volume approach may provide a sensitive alternative to standard thresholding techniques for capturing subtle functional changes in the architecture of FC in ASD.

Reduced amygdaloid vmPFC strength link our functional volume abnormalities to results showing under-connectivity patterns in ASD populations. As the vmPFC is part of the mentalizing or the theory of mind network, person perception, self-knowledge (Amodio and Frith. 2006) and the processing of pleasant outcomes like social and monetary rewards (Rademacher et al. 2010) our results align well with the idea that FC along the amygdala-vmPFC pathway might be altered in ASD. One study showed that the dorsal medial PFC is activated rather than the ventral medial PFC in ASD during a self-referential task, which suggests under-connectivity of the vmPFC in ASD (Schulte-Rüther et al. 2011). Another study found amygdalo-vmPFC underconnectivity when viewing sad faces in ASD (Swartz et al. 2013). Yet another study investigated oxytocin-induced activation, i.e. a crucial hormone in affective processing, in the vmPFC and pointed to an oxytocin-induced activation increase in the vmPFC and that this effect furthermore improved socio-communication difficulties in ASD (Aoki et al. 2015). Thus, our findings of decreased $\mathrm{FC}_{\mathrm{APF}-\mathrm{vmPFC}}$ strength are consistent with the known abnormalities along the amygdala-vmPFC pathway in ASD.

The amygdala is known to be a complex subcortical structure with many efferent and afferent subcortical and cortical as well as intra amygdala connections. Therefore, investigating amygdala functional connections using the entire 
amygdala does not account for its complex underlying pathways. One more fine grained method to investigate abnormal amygdala functional connections is to parcellate the amygdala based on anatomical subregions within the amygdala (Rausch et al. 2016; Ball et al. 2007; Roy et al. 2009) to assess subregion specific abnormalities. This method is however restricted to predefined anatomical ROI's based on healthy adult brains, which furthermore are associated to multiple functional pathways. In the current study, therefore we parcellated the amygdala into three functionally defined network parcels based on known amygdala SBNs within our own adolescent sample. This way, we established functionally meaningful amygdaloid subregions in our control sample, which were used to characterize the FC within three important amygdala SBNs in controls and our ASD group.

One potential limitation of our study is the use of a small homogenous sample. Our ASD sample does not include individuals with highly prevalent co-morbidities in ASD such as anxiety, depression or ADHD. No individuals with PDD-NOS were included in our sample. Therefore, our results only provide evidence for autistic core features. Although there was no trend towards group effects in $\mathrm{FC}_{\mathrm{AOF}-1 \mathrm{OFC}}(p=.682)$ or $\mathrm{FC}_{\mathrm{AAC}-\mathrm{cACC}}(p=.162)$ strength, future work involving larger ASD samples may be able to stratify the FC of the SBNs according to age, gender, and symptoms (Murphy and Spooren 2012). In addition, in order to maximize sensitivity of social measures for predicting functional volume, AQ measures may be complemented with interview data (Vineland) and observational measurements (ADOS), which could not be included in the present work as they were deemed too demanding for the ASD group who had already been diagnosed at the time of the study.

Our results demonstrate that functional amygdala parcellation based on its FC with three major amygdala SBNs is a sensitive measure for capturing the functional architecture of dysfunctional amygdalocortical pathways in ASD. Within the three SBNs that were investigated within this study, our results suggest that underconnectivity between amygdala and prefrontal vmPFC is driving abnormal functional interactions between the amygdala and other amygdala networks. By parcellating the amygdala functionally into volumes pathophysiological mechanisms along the amygdalo-prefrontal pathway could be linked to increasing symptom severity in ASD.

Acknowledgments The authors would like to thank Marieke W.M. Kuiper for her assistance with the data collection and Erik S.B. van Oort, Maarten Mennes, Erno J. Hermans, Marcel P. Zwiers for their support during preprocessing.

Funding This research was funded in part by Hersenstichting Fellowship [F2010(1)-13] awarded to W.B.G. The research leading to these results also received support from the European Community's Seventh Framework Programme (FP7/2007-2013) under grant agreement number 278948 (TACTICS), and the Innovative Medicines Initiative Joint Undertaking under grant agreement number 115300 (EU-AIMS), resources of which are composed of financial contribution from the European Union's Seventh Framework Programme (FP7/2007-2013) and the European Federation of Pharmaceutical Industries and Associations (EFPIA) companies' in kind contribution. C.F.B and K.V.H. are supported by the Netherlands Organisation for Scientific Research (NWO-Vidi 864-12-003 to C.F.B; NWO 016.Veni.171.068 to K.V.H) and further gratefully acknowledge funding from the Wellcome Trust UK Strategic Award [098369/Z/12/Z].

Author Contributions AR performed the measurements, performed the statistical analysis, participated to the interpretation and drafted the manuscript; $\mathrm{CB}$ participated in the design and interpretation of the data; JB participated in the design and interpretation of the data; $\mathrm{KH}$ participated in the design of the study, performed the statistical analysis, participated to the interpretation of the data and helped to draft the manuscript; WG conceived of the study, participated in its design, performed the measurements, participated to the interpretation of the data and helped to draft the manuscript. WZ performed the measurements, participated to the interpretation of the data and drafted the manuscript. All authors read and approved the final manuscript.

\section{Compliance with Ethical Standards}

Conflict of interest Christian F. Beckmann is director and shareholder of SBGneuro Ltd. Jan K Buitelaar has been in the past 3 years a consultant to/member of advisory board of/and/or speaker for Janssen Cilag BV, Eli Lilly, Lundbeck, Shire, Roche, Medice, Novartis, and Servier. He has received research support from Roche and Vifor. He is not an employee of any of these companies, and not a stock shareholder of any of these companies. He has no other financial or material support, including expert testimony, patents, royalties. Annika Rausch, Wei Zhang, Wouter B. Groen and Koen V. Haak declare that they have no competing interests.

Ethical Approval All procedures performed in studies involving human participants were in accordance with the ethical standards of the institutional and/or national research committee and with the 1964 Helsinki declaration and its later amendments or comparable ethical standards. This article does not contain any studies with animals performed by any of the authors.

Informed Consent Informed consent was obtained from all individual participants included in the study.

Open Access This article is distributed under the terms of the Creative Commons Attribution 4.0 International License (http://creativecommons.org/licenses/by/4.0/), which permits unrestricted use, distribution, and reproduction in any medium, provided you give appropriate credit to the original author(s) and the source, provide a link to the Creative Commons license, and indicate if changes were made.

\section{References}

American Psychiatric Association. (2000) Diagnostic and statistical manual of mental disorders: DSM-IV-TR ${ }^{\circledR}$. Washington, DC: American Psychiatric Publisher.

American Psychiatric Association. (2013) Diagnostic and statistical manual of mental disorders $\left(D S M-5^{\circledR}\right)$. Washington, DC: American Psychiatric Publisher. 
Amodio, D. M., \& Frith, C. D. (2006). Meeting of minds: The medial frontal cortex and social cognition. Nature Reviews Neuroscience, 7(4), 268-277.

Amunts, K., Kedo, O., Kindler, M., et al. (2005). Cytoarchitectonic mapping of the human amygdala, hippocampal region and entorhinal cortex: Intersubject variability and probability maps. Anatomy and Embryology, 210(5-6), 343-352.

Aoki, Y., Watanabe, T., Abe, O., et al. (2015). Oxytocin's neurochemical effects in the medial prefrontal cortex underlie recovery of task-specific brain activity in autism: A randomized controlled trial. Molecular Psychiatry, 20(4), 447-453.

Ball, T., Rahm, B., Eickhoff, S. B., Schulze-Bonhage, A., Speck, O., \& Mutschler, I. (2007). Response properties of human amygdala subregions: Evidence based on functional MRI combined with probabilistic anatomical maps. PLoS ONE, 2(3), e307.

Baron-Cohen, S., Ring, H. A., Bullmore, E. T., Wheelwright, S., Ashwin, C., \& Williams, S. C. (2000). The amygdala theory of autism. Neuroscience and Biobehavioral Reviews, 24(3), 355-364.

Baron-Cohen, S., Wheelwright, S., Skinner, R., Martin, J., \& Clubley, E. (2001). The autism-spectrum quotient (AQ): Evidence from asperger syndrome/high-functioning autism, malesand females, scientists and mathematicians. Journal of Autism and Developmental Disorders, 31(1), 5-17.

Bellani, M., Calderoni, S., Muratori, F., \& Brambilla, P. (2013). Brain anatomy of autism spectrum disorders II. Focus on amygdala. Epidemiology and Psychiatric Sciences, 22, 309-312.

Bickart, K. C., Dickerson, B. C., \& Barrett, L. F. (2014). The amygdala as a hub in brain networks that support social life. Neuropsychologia, 63, 235-248.

Bickart, K. C., Hollenbeck, M. C., Barrett, L. F., \& Dickerson, B. C. (2012). Intrinsic amygdala-cortical functional connectivity predicts social network size in humans. The Journal of Neuroscience, 32(42), 14729-14741.

Bickart, K. C., Wright, C. I., Dautoff, R. J., Dickerson, B. C., \& Barrett, L. F. (2010). Amygdala volume and social network size in humans. Nature Neuroscience, 14(2), 163-164.

Birn, R. M., Smith, M. A., Jones, T. B., \& Bandettini, P. A. (2008). The respiration response function: The temporal dynamics of fMRI signal fluctuations related to changes in respiration. NeuroImage, 40(2), 644-654.

Chang, C., Metzger, C. D., Glover, G. H., Duyn, J. H., Heinze, H. -J., \& Walter, M. (2013). Association between heart rate variability and fluctuations in resting-state functional connectivity. NeuroImage, 68, 93-104.

Gläscher, J., \& Adolphs, R. (2003). Processing of the arousal of subliminal and supraliminal emotional stimuli by the human amygdala. The Journal of Neuroscience: The Official Journal of the Society for Neuroscience, 23(32), 10274-10282.

Glover, G. H., Li, T. Q., \& Ress, D. (2000). Image-based method for retrospective correction of physiological motion effects in fMRI: RETROICOR. Magnetic Resonance in Medicine: Official Journal of the Society of Magnetic Resonance in Medicine/Society of Magnetic Resonance in Medicine, 44(1), 162-167.

Gorka, A. X., Torrisi, S., Shackman, A. J., Grillon, C., \& Ernst, M. (2017). Intrinsic functional connectivity of the central nucleus of the amygdala and bed nucleus of the stria terminalis. NeuroImage. (in press)

Green, S. A., Rudie, J. D., Colich, N. L., et al. (2013). Overreactive brain responses to sensory stimuli in youth with autism spectrum disorders. Journal of the American Academy of Child \& Adolescent Psychiatry, 52(11), 1158-1172.

Hahamy, A., Behrmann, M., \& Malach, R. (2015). The idiosyncratic brain: Distortion of spontaneous connectivity patterns in autism spectrum disorder. Nature Neuroscience, 18(2), 302-309.
Harms, M. B., Martin, A., \& Wallace, G. L. (2010). Facial emotion recognition in autism spectrum disorders: A review of behavioral and neuroimaging studies. Neuropsychology Review, 20(3), 290-322.

IBM Corp. (2016). IBM SPSS Statistics 23 step by step: A simple guide and reference. London: Routledge.

Kelly, R. E. Jr., Alexopoulos, G. S., Wang, Z., et al. (2010). Visual inspection of independent components: Defining a procedure for artifact removal from fMRI data. Journal of Neuroscience Methods, 189(2), 233-245.

Kort D. W., Schittekatte, M., Compaan, E., et al. (2002). Wisc-iii nl. Handleiding. Nederlandse bewerking. London: The Psychological Corporation.

Lord, C., Rutter, M., \& Le Couteur, A. (1994). Autism Diagnostic Interview-Revised: A revised version of a diagnostic interview for caregivers of individuals with possible pervasive developmental disorders. Journal of Autism and Developmental Disorders, 24(5), 659-685.

McMenamin, B. W., \& Marsolek, C. J. (2013). Can theories of visual representation help to explain asymmetries in amygdala function? Cognitive, Affective, \& Behavioral Neuroscience, 13(2), 211-224.

Motzkin, J. C., Philippi, C. L., Wolf, R. C., Baskaya, M. K., \& Koenigs, M. (2015). Ventromedial prefrontal cortex is critical for the regulation of amygdala activity in humans. Biological Psychiatry, 77(3), 276-284.

Murphy, D., \& Spooren, W. (2012). EU-AIMS: A boost to autism research. Nature Reviews. Drug discovery, 11(11), 815.

O'reilly, J. X., Beckmann, C. F., Tomassini, V., Ramnani, N., \& Johansen-Berg, H. (2009). Distinct and overlapping functional zones in the cerebellum defined by resting state functional connectivity. Cerebral Cortex, 20(4), 953-965.

Oldfield, R. C. (1971). The assessment and analysis of handedness: The Edinburgh inventory. Neuropsychologia, 9(1), 97-113.

Phelps, E. A., \& LeDoux, J. E. (2005). Contributions of the amygdala to emotion processing: From animal models to human behavior. Neuron, 48(2), 175.

Poser, B. A., Versluis, M. J., Hoogduin, J. M., \& Norris, D. G. (2006). BOLD contrast sensitivity enhancement and artifact reduction with multiecho EPI: Parallel-acquired inhomogeneity-desensitized fMRI. Magnetic Resonance in Medicine: Official Journal of the Society of Magnetic Resonance in Medicine/Society of Magnetic Resonance in Medicine, 55(6), 1227-1235.

Power, J. D., Barnes, K. A., Snyder, A. Z., Schlaggar, B. L., \& Petersen, S. E. (2012). Spurious but systematic correlations in functional connectivity MRI networks arise from subject motion. NeuroImage, 59(3), 2142-2154.

Rademacher, L., Krach, S., Kohls, G., Irmak, A., Gründer, G., \& Spreckelmeyer, K. N. (2010). Dissociation of neural networks for anticipation and consumption of monetary and social rewards. NeuroImage, 49(4), 3276-3285.

Rausch, A., Zhang, W., Haak, K. V., Mennes, M., Hermans, E. J., van Oort, E., van Wingen, G., Beckmann, C. F., Buitelaar, J. K., Groen, W. B. (2016). Altered functional connectivity of the amygdaloid input nuclei in adolescents and young adults with autism spectrum disorder: A resting state fMRI study. Molecular Autism, $7(1), 13$.

Rosenfeld, A. (1976). Digital picture processing. New York: Academic press.

Roy, A. K., Shehzad, Z., Margulies, D. S., et al. (2009). Functional connectivity of the human amygdala using resting state fMRI. NeuroImage, 45(2), 614.

Schulte-Rüther, M., Greimel, E., Markowitsch, H. J., et al. (2011). Dysfunctions in brain networks supporting empathy: An fMRI study in adults with autism spectrum disorders. Social Neuroscience, 6(1), 1-21. 
Smith, S. M., Jenkinson, M., Woolrich, M. W., et al. (2004). Advances in functional and structural MR image analysis and implementation as FSL. NeuroImage, 23, S208-S219.

Swartz, J. R., Wiggins, J. L., Carrasco, M., Lord, C., \& Monk, C. S. (2013). Amygdala habituation and prefrontal functional connectivity in youth with autism spectrum disorders. Journal of the American Academy of Child \& Adolescent Psychiatry, 52(1), 84-93.

Wechsler, D. (2000). WAIS-III Nederlandstalige bewerking: Afnameen scoringshandleiding [WAIS-III Dutch version: User manual]. 